\title{
Photoacoustic microscopy with submicron resolution
}

Konstantin Maslov, Geng Ku, Lihong V. Wang

Konstantin Maslov, Geng Ku, Lihong V. Wang, "Photoacoustic microscopy with submicron resolution," Proc. SPIE 7564, Photons Plus Ultrasound: Imaging and Sensing 2010, 75640W (23 February 2010); doi: 10.1117/12.841919

SPIE. Event: SPIE BiOS, 2010, San Francisco, California, United States 


\title{
Photoacoustic microscopy with submicron resolution
}

\author{
Konstantin Maslov, Geng Ku, and Lihong V. Wang \\ Optical Imaging Laboratory, Department of Biomedical Engineering, \\ Washington University in St. Louis, St. Louis, Missouri 63130
}

\begin{abstract}
We show that it is possible to obtain high optical contrast photoacoustic images of tissue with $0.55 \mu \mathrm{m}$ transverse resolution. To achieve high sensitivity, we used a high NA (0.85), $125 \mathrm{MHz}$ spherically focused ultrasonic transducer in a confocal arrangement with a high resolution optical objective (NA=0.6). Laser pulses of a few $\mathrm{nJ}$ in pulse energy with durations of $1.5 \mathrm{~ns}$ at a $20 \mathrm{KHz}$ pulse repetition rate were used to generate photoacoustic waves. Although the penetration depth is limited to hundreds of microns by both optical scattering and ultrasonic absorption, the developed technique can compete with optical microscopy, for example, in quantitative spectral measurements, in microcirculation research, or in nanoparticle detection.
\end{abstract}

Keywords: Photoacoustic imaging, photoacoustic microscopy, functional imaging, microcirculation, noninvasive imaging, longitudinal studies.

\section{INTRODUCTION}

In many potential clinical applications it is necessary to image anatomic structures with high resolution, to identify structures according to their optical spectral properties, or to detect very small biomarkers such as conjugated nanoparticles. There is also a significant amount of research performed in vivo on animal models, such as studies of angiogenesis or cancer metastatic activity by monitoring of circulated cancer cells. In such research a minimally invasive imaging technique suitable for longitudinal studies with at least the cellular resolution is required.

An emerging high-resolution imaging modality, photoacoustic microscopy (PAM) images optically absorbing microstructures by detecting transient acoustic waves generated from laser-induced thermal-elastic expansion. PAM is directly sensitive to optical absorption, which is a quantitative measure of some physiologically important endogenous molecules (e.g., hemoglobin and melanin) as well as a measure of various widely used optical contrast agents (e.g., methylene blue and indocyanine green). In acoustic-resolution PAM systems, both the transverse and axial resolutions are defined by ultrasonic detection. However, because of the overwhelming attenuation of high-frequency ultrasound beyond $100 \mathrm{MHz}$ in biological tissue, it is difficult to achieve a transverse resolution below $10 \mu \mathrm{m}$ with ultrasonic focusing without severely compromising the imaging depth. To overcome this obstacle, our group recently proposed optical-resolution PAM, whose transverse resolution is defined by a tight optical focus. Using an optical microscope objective with an NA of $0.1,5-\mu \mathrm{m}$ transverse resolution was experimentally obtained, and microvasculature was imaged down to the single-capillary level [1]. Here we extend the resolution of PAM to the submicron level for the first time.

The lateral resolution of photoacoustic microscopy is determined by its ability to focus light or ultrasound, through either device focusing or image reconstruction (somewhat equivalent to synthetic focusing). Consequently, the lateral resolution can theoretically be made comparable to that of conventional optical microscopy. However, the possibility of detecting optical absorption in such a small volume is a challenging problem because the small source of the photoacoustic wave generates a small amount of acoustic power, which must exceed the noise level to be effectively detected.

Photons Plus Ultrasound: Imaging and Sensing 2010, edited by Alexander A. Oraevsky, Lihong V. Wang, Proc. of SPIE Vol. 7564, 75640W · (C) 2010 SPIE - CCC code: 1605-7422/10/\$18 - doi: 10.1117/12.841919 
Let us consider a small optically absorbing volume, $V$, occupying the focal area of the objective. Due to its small dimension in comparison to the wavelength of the detected acoustic wave, the photoacoustic source can be approximated by a monopole (point) source of ultrasound. The peak power of the generated acoustic wave can be easily calculated [2]. At a distance $r$, the photoacoustic pressure generated by a small optically absorptive object illuminated by a laser pulse of a Gaussian shape $I=\frac{1}{\sqrt{\pi}} \frac{F_{0}}{\tau} \exp \left(-(t / \tau)^{2}\right)$ can be expressed as [2]:

$$
p=\frac{\sigma \beta F}{2 \pi \tau^{2} C_{p} r}\left[\frac{-2 t}{\tau} \exp \left(-(t / \tau)^{2}\right)\right]
$$

where $F_{0}$ is the optical energy fluence, $\beta$ is the isobaric volume expansion coefficient, $\sigma$ is the optical absorption cross section of the absorbing volume, and $C_{\mathrm{p}}$ is the specific heat. For an absorption coefficient, $\mu_{\mathrm{a}}$, whose reciprocal is small in comparison to the size of the absorbing volume, $\sigma \approx \mu_{a} V$. Equation (1) can be used to calculate the peak power of the photoacoustic wave on an ultrasonic transducer with a numerical aperture $N A=0.85$ :

$$
W_{\max } \approx \frac{4}{\pi e} \frac{1}{\rho c}\left(\frac{\beta \mu_{a} F V}{C_{p} \tau^{2}}\right)^{2} \cdot \frac{N A^{2}}{2} \approx 0.5 \cdot 10^{-7} \mathrm{~W},
$$

where $\rho$ is mass density and $c$ is the speed of sound. Here we insert in (2) realistic parameters: the optical absorption coefficient of red blood cells (RBC) $\mu_{a} \sim 750 \mathrm{~cm}^{-1}$, focal volume $V \sim 1 \mu \mathrm{m}^{3}$, which corresponds to an optical resolution of approximately $0.5 \mu \mathrm{m}$, and $F_{0}=20 \mathrm{~mJ} / \mathrm{cm}^{2}$ which is the ANSI safety limit for unintended exposure of the human skin [3]. The full width at half maximum (FWHM) pulse width of the laser pulse was $1.5 \mathrm{~ns}$ which gives $\tau=F W H M / 2 \sqrt{\ln 2} \cdot \sim 1 n s$. As one can see, the calculated peak power of the photoacoustic signal is well above the thermal nose level of ideally electrically matched transducer:

$$
W_{n}=k T \Delta f=2.5 \cdot 10^{-12} \mathrm{~W}, ।
$$

where $k$ is the Boltzmann constant, $T=300 K$ is the ambient temperature, and $\Delta f=0.5 \cdot 10^{9}$ is the signal frequency band. Taking into account transducer losses, which for broad-band transducers typically exceed $20 \mathrm{~dB}$, and ultrasonic absorption in the tissue and immersion liquid, we still conclude that submicron resolution imaging using PAM is feasible. However, to achieve a reasonable signal to noise ratio (SNR) without signal averaging, the optical fluence should be hundreds of millijoules per square centimeter. Although ANSI does not regulate fluence level in the focal area of a tightly focused beam, it sets the safety limit for skin exposure for a collimated laser beam at $20 \mathrm{~mJ} / \mathrm{cm}^{2}$. The ANSI limit notwithstanding, previously published works [4,5] have put the safe exposure limit for RBCs to $0.5 \mathrm{~J} / \mathrm{cm}^{2}$ and even higher for other types of cells. That level of fluence should be enough to achieve an SNR of at least around $30 \mathrm{~dB}$.

\section{METHODS AND MATERIALS}

The schematic of the OR-PAM system is presented in Figure 1. The target tissue was irradiated by a short-pulsed laser beam. Wideband ultrasonic waves (referred to as photoacoustic waves) were induced as a result of transient thermoelastic expansion due to the tissue's absorption of optical energy. Our system employed optical focusing using a long working distance high resolution optical objective (Zeiss Plan 40/0.60) to achieve $\mu$ m-level lateral resolution. A $\mathrm{Nd}: \mathrm{YVO}_{4}$ laser (Elforlight, UK) was used as the irradiation source. Laser pulses of $1.5 \mathrm{~ns}$ durations were coupled to a single-mode optical fiber, the output of which was imaged to a diffraction-limited focal spot within the sample volume. The laser pulse energy after the objective lens was measured to be $40 \mathrm{~nJ}$. Ultrasonic focusing was achieved through a concave lens (radius of curvature: $1.8 \mathrm{~mm}$; aperture: $3.2 \mathrm{~mm}$; NA in water: 0.85 ) ground into the end of the quartz waveguide of the $125 \mathrm{MHz}$ central frequency broad band ultrasonic transducer (Panametrics V2062). The optical objective lens and the ultrasonic transducer were configured coaxially and confocally as shown in Fig. 1. The object under investigation (e.g., cell culture, mouse ear, and tissue slice) was placed on a microscope slide and covered with an ultrasonically and optically transparent polyethylene membrane. Ultrasonic gel (Clear Image, SonoTech, Bellingham, 
WA) was applied between the polyethylene membrane and the animal tissue for acoustic coupling. The photoacoustic signal detected by the ultrasonic transducer was amplified by two cascaded amplifiers (ZX60-3018G-S and ZFL 500LN, Mini-Circuits,) and then was digitized by a data acquisition (DAQ) board (NI PCI-5154) at a sampling rate of 2 GS/s. Along the transverse $(x-y)$ plane, two dimensional (2D) raster scanning of the confocally positioned optical objective and ultrasonic transducer together was controlled by a separate PC, which triggered both the DAQ board and the pump laser. Axial (z-axis) position of the focal spot was controlled by a piezoelectric actuator (PAS100, Thorlabs). A transmissionmode optical microscope was integrated into our system by moving a light-emitting diode into the optical path and replacing the ultrasonic transducer with an optical microscope. This addition helps us quickly target the same region of the animal for multi-day monitoring.

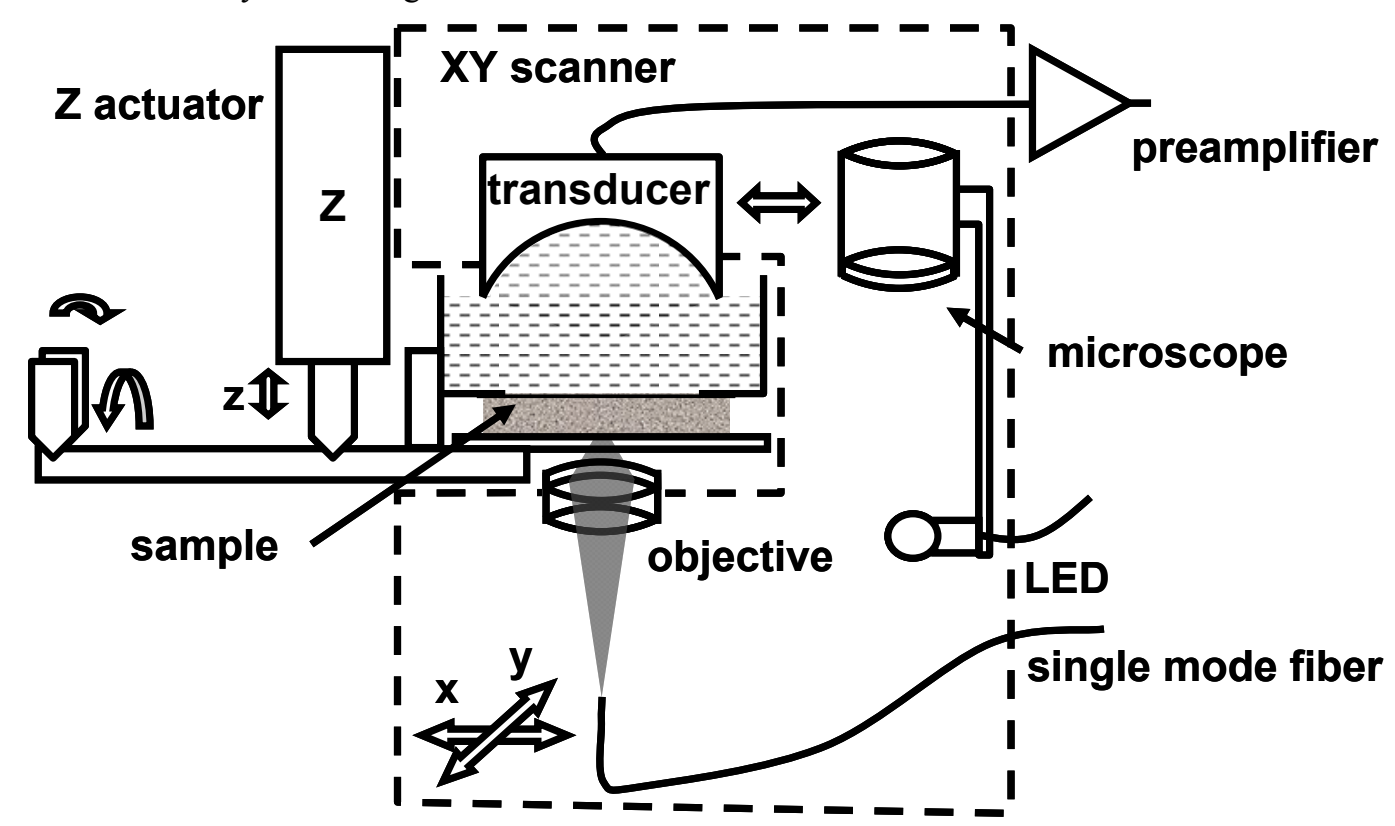

Fig. 1. Experimental setup.

Swiss-Webster mice (Hsd:ND4, Harlan Co.; body weight, $\sim 30 \mathrm{~g}$ ) were imaged. Before imaging, the hair on the ears was gently removed with human hair-removing lotion (Surgi Cream, Ardell Int'l). Mice were anesthetized using 1.0-1.5\% isoflurane and transferred to a custom-made stereotactic stage. Throughout the experiment, anesthesia was maintained using $1.0-1.5 \%$ isoflurane with an airflow rate of $1 \mathrm{~L} / \mathrm{min}$, and the body temperature of the animal was maintained at $37^{\circ} \mathrm{C}$ with a temperature controlled heating pad. At the end of the study, mice were euthanized by pentobarbital, 100 $\mathrm{mg} / \mathrm{kg}$, i.p. All animal care and experimental procedures were carried out in conformance with the laboratory animal protocol approved by the School of Medicine Animal Studies Committee of Washington University in St. Louis.

\section{RESULTS}

In submicron PAM, the transverse resolution is limited by the radial size of the optical focal zone. An ideal objective lens focuses a plane wave to a diffraction-limited Airy pattern, whose size is determined only by the NA and the optical wavelength. Given the aforementioned system parameters, the blurring of a point absorber can be predicted by the FWHM of the Airy disk, which is $0.45 \mu \mathrm{m}$ for a FWHM $=0.51 \lambda / \mathrm{NA}$. To test the PAM system, we imaged a gelatin sample mixed with gold nanocages, a promising contrast agent for photoacoustic imaging, at a low concentration. The gold nanocages had a typical size of $150 \mathrm{~nm}$, and thus could be approximately considered as point absorbers here. The transverse profile of a typical nanocage image is shown in Fig. 2a. Interestingly enough, the transverse spread function of the system measured this way is narrower than the theoretical FWHM of $0.45 \mu \mathrm{m}$. However, this result was expected as being caused by the extremely high ratio of absorption cross section of the nanocage to nanocage volume and to the nonlinearity of the thermal expansion coefficient. As has been theoretically shown before [2], in this case the photoacoustic signal becomes proportional to at least the second power of the optical fluence, which effectively narrows the transverse profile of the image of the nanoparticle. 


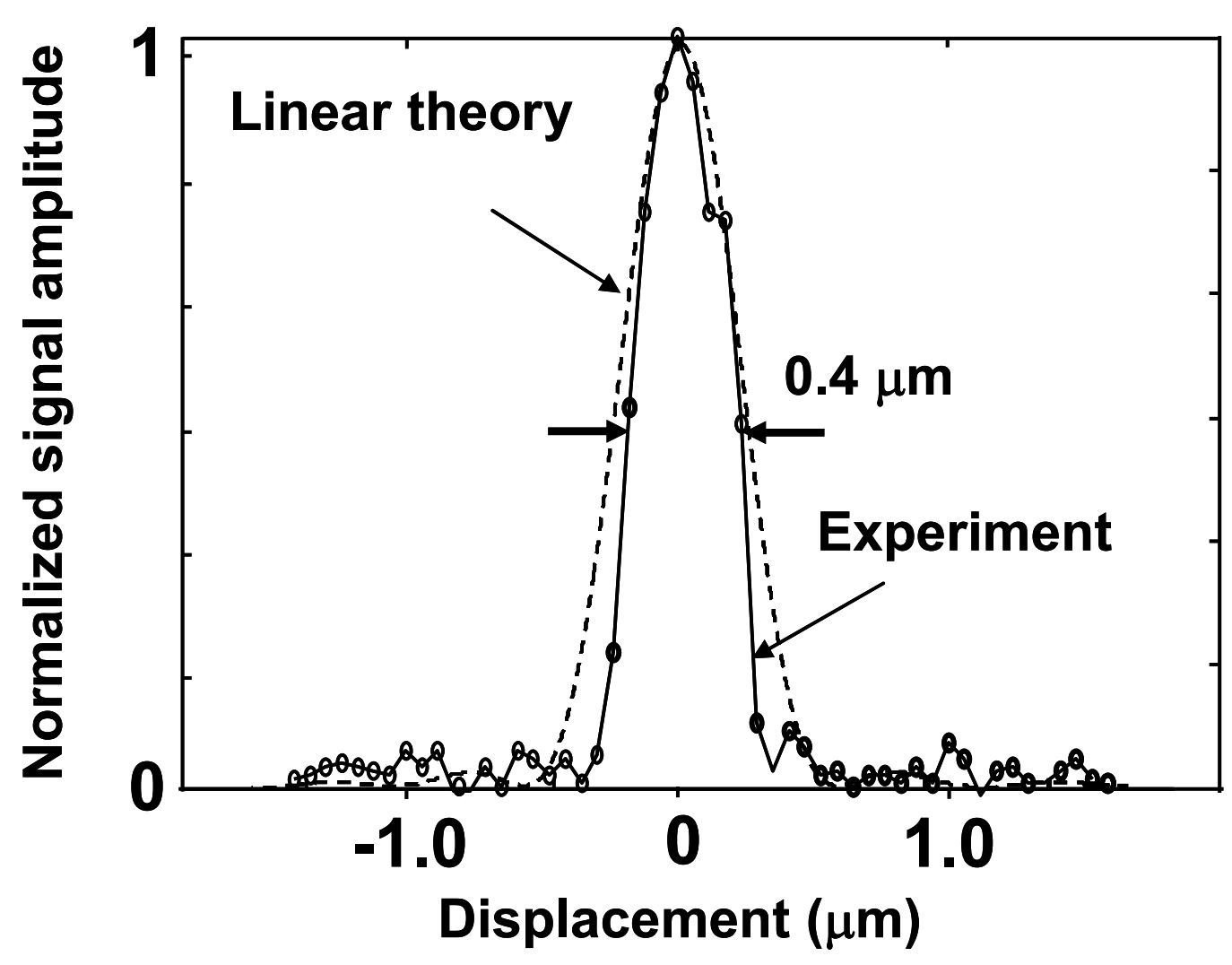

Fig. 2 Transverse spread profile of a $150 \mu \mathrm{m}$ diameter nanocage. Circle: Experimental data. Dashed line: Theoretical intensity distribution for an ideal lens with $\mathrm{NA}=0.6$.

We employed the submicron PAM to image a Swiss-Webster mouse ear in vivo. The mouse ear is a widely used model for studying cutaneous microcirculation [6]. As a predominant endogenous light absorber, hemoglobin enables photoacoustic imaging of the microcirculation without angiographic contrast agents. Thus, blood vessels to be detected by label-free photoacoustic imaging must contain RBCs, the carriers of hemoglobin. This unique characteristic allows photoacoustic microscopy to image only RBC-perfused capillaries, the functional capillary subset supplying oxygen to living tissues.

In the present study, the optical wavelength was $532 \mathrm{~nm}$, which is close to an isosbestic point of the hemoglobin absorption spectrum. Thus, the detected photoacoustic signal reflected the volumetric distribution of the total hemoglobin concentration, regardless of the blood oxygen level.

Figure 3a, a maximum amplitude projection (MAP) image of a $1 \mathrm{~mm} \times 1 \mathrm{~mm}$ area of a mouse ear shows that PAM was able to depict the detailed morphology of the microvasculature. More interestingly, in Fig. 3b, which is an image of a smaller fragment of the same ear, we also observed a large number of "dots", and most of them appeared to be aligned along curved lines. A close-up view of a small part of Fig 3b, designated by a dashed box, is shown in detail in Fig. 3d. From the characteristic donut shape of the "dots" one can see that they are most plausibly motionless RBCs in capillaries where blood flow was arrested. At such high resolution, the capillary image is non-uniform, as seen in Fig. 3c, because PAM images show high contrast objects such as RBCs, but not capillary walls. Correspondingly, when scanning along a capillary, the number of RBCs crossed by the laser beam depends on the distance between the RBCs and the relative speeds of the laser beam and blood. By comparing two consecutive image lines taken using bidirectional scanning, it is possible to measure blood stream velocity in capillaries. 


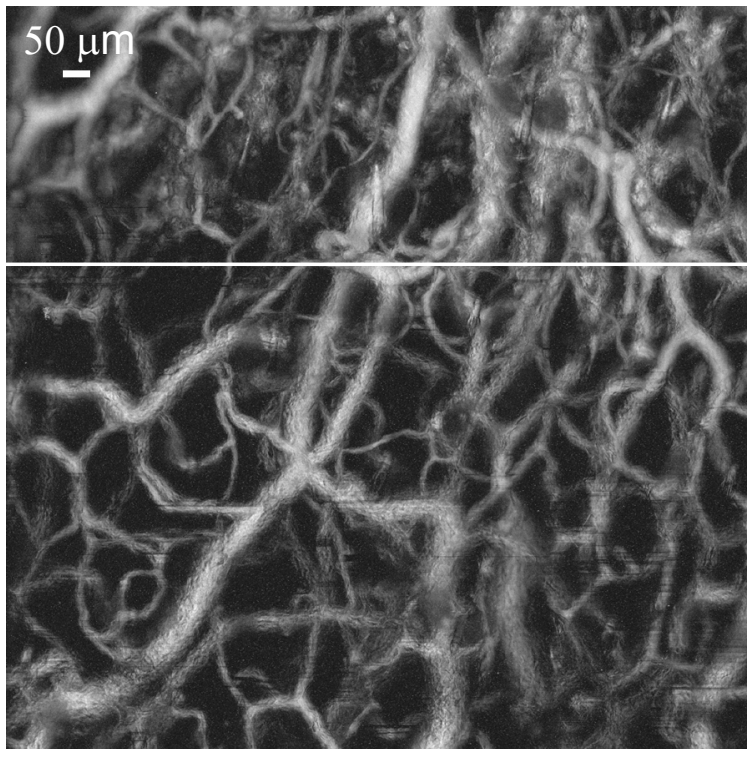

(a)

(b)

(c)
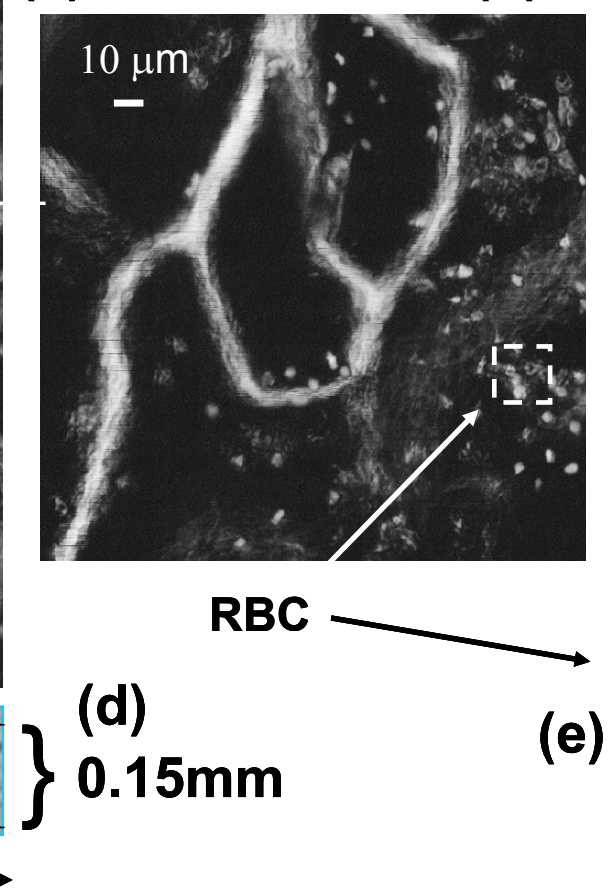

(e)
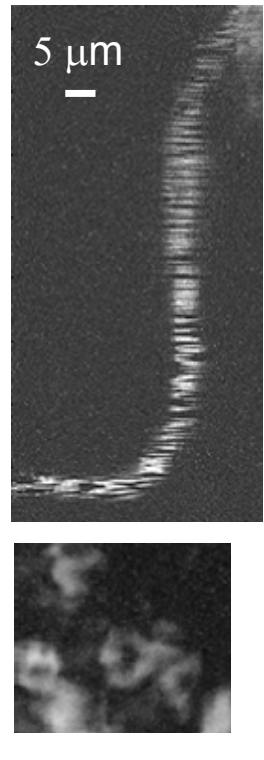

$1 \mathrm{~mm}$

Fig. 3 Photoacoustic image of a mouse ear. (a) Maximum amplitude projection image of a $1 \mathrm{~mm} \times 1 \mathrm{~mm}$ area of the mouse ear, (b) image at a higher magnification, (c) single capillary in mouse ear, (d) cross sectional B-scan image along the line shown in (a), and (e) close-up of the area indicated by the box in (b).

A photoacoustic dataset carries three-dimensional information $(x-y$ for mechanical scanning and $z$ for arrival time of photoacoustic signals). From Eq. (2) it follows that for a large absorber the PAM signal amplitude should not depend on the absorber's position along the $z$ axis, providing that the light beam is located within the focus area of the ultrasonic transducer. Correspondingly, out of focal plane blood vessels must be as bright as vessels located in the focal plane, albeit blurred laterally. To illustrate this effect, a cross sectional (B-scan) image corresponding to a scanning line designated by a white line in Fig. 3a is shown in Fig. 3d. The PAM was focused to the depth of $150 \mu \mathrm{m}$ inside a mouse ear. Figure $3 \mathrm{~d}$ shows that capillaries remain well resolved at $150 \mu \mathrm{m}$ depth. The vessels seen in the upper part of Fig. 3a, which are $\sim 250 \mu \mathrm{m}$ from the surface, appear not only blurred but also distorted, which is caused by an optically inhomogeneous cartilage layer lying between the optical objective and the imaging plane. Hence the imaging depth with preserved near-micron resolution is about 100 to $150 \mu \mathrm{m}$ in a mouse ear.

In summary, we have developed a new generation of optical-resolution photoacoustic microscopy that has a submicron transverse resolution of $\sim 0.5 \mu \mathrm{m}$, which is comparable to the resolution of conventional optical microscopy. The axial resolution was estimated to be $\sim 10 \mu \mathrm{m}$, as defined by the bandwidth of the ultrasonic transducer, and the maximum penetration depth at $<1 \mu \mathrm{m}$ transverse resolution was tested to be about $150 \mu \mathrm{m}$ in the mouse skin. In vivo studies on a mouse ear showed that it is feasible to visualize individual red blood cells in micro-vessels. The submicron resolution PAM was used to repeatedly image the same area in a mouse ear during a course of 30 days. No visible difference in vessel structure was noticed, indicating that PAM is well suited for longitudinal studies. The transverse resolution can be improved by employing an even higher NA objective lens and carefully controlling optical aberrations. With future improvements in imaging speed, we expect that this new imaging technology will find broad in vivo applications in quantifying capillary flow, oxygenation, and potentially the metabolic rate of oxygen at the single-cell level, and will provide new insight into microcirculation studies. 


\section{ACKNOWLEDGEMENT}

This project is sponsored in part by National Institutes of Health Grant Nos. R01 EB000712, R01 EB008085, R01 CA113453901, U54 CA136398, 5P60 DK02057933 L.W. has a financial interest in Microphotoacoustics, Inc. and Endra, Inc., which, however, did not support this work. Additional information about related works can be found at http://oilab.seas.wustl.edu.

\section{REFERENCES}

[1] K. Maslov, H. F. Zhang, S. Hu, and L. V. Wang, "Optical-resolution photoacoustic microscopy for in vivo imaging of single capillaries," Opt. Lett. 33(9), 929-931 (2008).

[2] I. G. Calasso, W. Craig, and G. J. Diebold, "Photoacoustic Point Source" Phys.l Rev. Lett. 86(16), 3550-3553 (2001).

[3] "American national standard for the safe use of lasers Z136.1," American National Standards Institute, New York, (2000).

[4] V. P. Zharov, E. I. Galanzha, E. V. Shashkov, N. G. Khlebtsov, and V. V. Tuchin, "In vivo photoacoustic flow cytometry for monitoring of circulating single cancer cells and contrast agents," Optics Let. 31(24), 3623-3625 (2006).

[5] D. O. Lapotko, and V. P. Zharov, "Spectral Evaluation of Laser-Induced Cell Damage With Photothermal Microscopy," Lasers Surg. Med. 36(1), 22-30 (2005)

[6] J. H. Barker, F. Hammersen, I. Bondar, E. Uhl, T. J. Galla, M. D. Menger, and K. Messmer, "The hairless mouse ear for in vivo studies of skin microcirculation," Plast. Reconstr. Surg. 83(6), 948-959 (1989) 